\title{
Cues and control in Expert-Client Dialogues
}

\author{
Steve Whittaker \& Phil Stenton \\ Hewlett-Packard Laboratories \\ Filton Road, Bristol BS12 6QZ, UK. \\ email: sjw@hplb.csnet
}

April 18, 1988

\begin{abstract}
We conducted an empirical analysis into the relation between control and discourse structure. We applied control criteria to four dialogues and identified 3 levels of discourse structure. We investigated the mechanism for changing control between these structures and found that utterance type and not cue words predicted shifts of control. Participants used certain types of signals when discourse goals were proceeding successfully but resorted to interruptions when they were not.
\end{abstract}

\section{Introduction}

A number of researchers have shown that there is organisation in discourse above the level of the individual utterance $(5,8,9,10)$, The current exploratory study uses control as a parameter for identifying these higher level structures. We then go on to address how conversational participants co-ordinate moves between these higher level units, in particular looking at the ways they use to signal the beginning and end of such high level units.

Previous research has identified three means by which speakers signal information about discourse structure to listeners: Cue words and phrases $(5,10)$; Intonation (7); Pronomi- nalisation $(6,2)$. In the cue words approach, Reichman (10) has claimed that phrases like "because", "so", and "but" offer explicit information to listeners about how the speaker's current contribution to the discourse relates to what has gone previously. For example a speaker might use the expression "so" to signal that $s /$ he is about to conclude what $s /$ he has just said. Grosz and Sidner (5) relate the use of such phrases to changes in attentional state. An example would be that "and" or "but" signal to the listener that a new topic and set of referents is being introduced whereas "anyway" and "in any case" indicate a return to a previous topic and referent set. A second indirect way of signalling discourse structure is intonation. Hirschberg and Pier rehumbert (7) showed that intonational contour is closely related to discourse segmentation with new topics being signalled by changes in intonational contour. A final more indirect cue to discourse structure is the speaker's choice of referring expressions and grammatical structure. A number of researchers $(4,2,6,10)$ have given accounts of how these relate to the continuing, retaining or shifting of focus.

The above approaches have concentrated on particular surface linguistic phenomena and then investigated what a putative cue serves to signal in a number of dialogues. The problem 
with this approach is that the cue may only be an infrequent indicator of a particular type of shift. If we want to construct a general theory of discourse than we want to know about the whole range of cues serving this function. This study therefore takes a different approach. We begin by identifying all shifts of control in the dialogue and then look at how each shift was signalled by the speakers. A second problem with previous research is that the criteria for identifying discourse structure are not always made explicit. In this study explicit criteris are given: we then go on to analyse the relation between cues and this structure.

\section{The data}

The data were recordings of telephone conversations between clients and an expert concerning problems with software. The tape recordings from four dialogues were then transcribed and the analysis conducted on the typewritten transcripts rather than the raw recordings. There was a total of 450 turns in the dialogues.

2.1 Criteria for classifying utterance types. Each utterance in the dialogue was classified into one of four categories: (a) Assertions - declarative utterances which were used to state facts. Yes or no answers to questions were also classified as assertions on the grounds that they were supplying the listener with factual information; (b) Commands utterances which were intended to instigate action in their audience. These included various utterances which did not have imperative form, (e.g. "What I would do if I were you is to relink $X^{\prime}$ ) but were intended to induce some action; (c) Questions - utterances which were intended to elicit information from the audience. These included utterances which did not have interrogative form. e.g. "So my question is...." They also included paraphrases, in which the speaker reformulated or repeated part or all of what had just been said. Paraphrases were classified as questions on the grounds that the effect was to induce the listener to confirm or deny what had just been stated; (d) Prompts - These were utterances which did not express propositional content. Examples of prompts were things like "Yes" and "Uhu".

2.2 Allocation of control in the dialogues. We devised several rules to determine the location of control in the dialogues. Each of these rules related control to utterance type: (a) For questions, the speaker was defined as being in control unless the question directly followed a question or command by the other conversant. The reason for this is that questions uttered following questions or commands are normally attempts to clarify the preceding utterance and as such are elicited by the previous speaker's utterance rather than directing the conversation in their own right. (b) For assertions, the speaker was defined as being in control unless the assertion was made in response to a question, for the same reasons as those given for questions; an assertion which is a response to a question could not be said to be controlling the discourse; (c) For commands, the speaker was defined as controlling the conversation. Indirect commands (i.e. utterances which did not have imperative form but served to elicit some actions) were also classified in this way; (d) For prompts, the listener was defined as controlling the conversation, as the speaker was clearly abdicating his/her turn. In cases where a turn consisted of several utterances, the control rules were only applied to the final utterance.

We applied the control rules and found that control did not alternate from speaker to speaker on a turn by turn basis, but that there were long sequences of turns in which control remained with one speaker. This seemed to suggest that the dialogues were organised above the level of individual turns into phases 
where control was located with one spesker. The mean number of turns in each phase was 6.63.

\section{Mechanisms for switch- ing control}

We then went on to analyse how control was exchanged between participants at the boundaries of these phases. We first examined the last utterance of each phase on the grounds that one mechanism for indicating the end of a phase would be for the speaker controlling the phase to give some cue that he (both participants in the dialogues were always male) no longer wished to control the discourse. There was a total of 56 shifts of control over the 4 dialogues and we identified 3 main classes of cues used to signal control shifts These were prompts, repetitions and summaries. We also looked at when no signal was given (interruptions).

3.1 Prompts. On 21 of the 56 shifts (38\%), the utterance immediately prior to the control shift was a prompt. We might therefore explain these shifts as resulting from the person in control explicitly indicating that he had nothing more to say.

(In the following examples a line indicates a control shift)

Example 1 - Prompt Dialogue C -

1. E: "And they are, in your gen you'll find that they've relocated into the labelled common area" (E control)

2. C: "That's right." (E control)

3. E: "Yeah" (E abdicates control with prompt)
4. C: "I've got two in there. There are two of them." (C control)

5. E: "Right" (C control)

6. C: "And there's another one which is \% RESA" ( $\mathrm{C}$ control)

7. E: "OK um" (C control)

8. C: "VS" (C control)

9. E: "Right" (C control)

10. C: "Mm" (C abdicates control with prompt)

11. E: "Right and you haven't got - I assume you haven't got local labelled common with those labels" ( $\mathrm{E}$ control)

3.2 Repetitions and summaries. On a further 15 occasions ( $27 \%$ ), we found that the person in control of the dialogue signalled that they had no new information to offer. They did this either by repeating what had just been said (6 occasions), or by giving a summary of what they had said in the preceding utterances of the phase ( 9 occasions). We defined a repetition as an assertion which expresses part or all of the propositional content of a previous assertion but which contains no new information. A summary consisted of concise reference to the entire set of information given about the client's problem or the solution plan.

Example 2 - Repetition. Dialogue C -

1. Client: "These routines are filed as $\mathrm{DS}^{\prime \prime}$ ( $\mathrm{C}$ control) 
2. Expert: "That's right, yes" (C control)

3. C: "DS" (C abdicates control with repetition)

4. E: "And they are, in your gen you'll find they've relocated

into your local common area." (E control)

Half the repetitions were accompanied by cue words. These were "and", "well" and "so", which prefixed the assertion.

Example 3 - Summary Dialogue B -

1. E. "OK. Initialise the disc retaining spares" ( $E$ control)

2. C: "Right" (E control)

3. E: "Uh and then TF it back" (E control)

4. C: "Right" (E control)

5. E: "Did you do the TF with verify?" ( $\mathrm{E}$ control)

6. C: "Er yes I did" (E control)

7. E: "OK. That would be my recommendation and that will ensure that you get er a logically integral set of files" ( $E$ abdicates control with summary)

8. C: "Right. You think that initialising it using this um EXER facility." (C control)
What are the linguistic characteristics of summaries? Reichman (10) suggests that "so" might be a summary cue on the part of the speaker but we found only one example of this, although there were 3 instances of "and", one "now" one "but" and one "so". In our dialogues the summaries seemed to be characterised by the concise reference to objects or entities which had earlier been described in detail, e.g. (a) "Now, I'm wondering how the two are related" in which "the two" refers to the two error messages which it had taken several utterances to describe previously. The other characteristic of summaries is that they contrast strongly with the extremely concrete descriptions elsewhere in the dialogues, e.g. "err the system program standard call file doesn't complete this means that the file does not have a tail record" followed by "And I've no clue at all how to get out of the situation". Example 3 also illustrates this change from specific $(1,3,5)$ to general $(7)$. How then do repetitions and summaries operate as cues? In summarising, the speaker is indicating a natural breakpoint in the dialogue and they also indicate that they have nothing more to add at that stage. Repetitions seem to work in a similar way: the fact that a speaker reiterates indicates that he has nothing more to say on a topic.

3.3 Interruptions. In the previous cases, the person controlling the dialogue gave a signal that control might be exchanged. There were 20 further occasions ( $36 \%$ of shifts) on which no such indication is given. We therefore went on to analyse the conditions in which such interruptions occurred. These seem to fall into 3 categories: (a) vital facts; (b) responses to vital facts; (c) clarifications.

3.3.1 Vital facts. On a total of 6 occasions (11\% of shifts) the client interrupted to contradict the speaker or to supply what seemed to be relevant information that he believed the expert did not know. 
Example 4 Dialogue C -

1. E: ".... and it generates this warning, which is now at 4.0 to warn you about the situation" (E control)

2. C: "It is something new though um" (C assumes control by interruption)

\section{E: "Well" (C control)}

4. C: "The programs that I've run before obviously LINK A's got some new features in it which er..." (C control)

5. E: "That's right, it's a new warning at 4.0" ( $E$ assumes control by interruption)

Two of these 6 interjections were to supply extra information and one was marked with the cue "as well". The other four were to contradict what had just been said and two had explicit markers "though" and "well actually": the remaining two being direct denials.

3.3.2 Reversions of control following vital facts. The next class of interruptions occur after the client has made some interjection to supply a missing fact or when the client has blocked a plan or rejected an explanation that the expert has produced. There were 8 such occasions ( $14 \%$ of shifts).

The interruption in the previous example illustrates the reversion of control to the expert after the client has supplied information which he (the client) believes to be highly relevant to the expert. In the following example, the client is already in control.

Example 5 Dialogue B -
1. "I'll take a backup first as you say" (C control)

2. E: "OK" (C control)

3. C: "The trouble is that it takes a long time doing all this" (C control)

4. E: "Yeah, yeah but er this kind of thing there's no point taking any short cuts or you could end up with no system at all." (E assumes control by interruption)

On five occasions the expert explicitly signified his acceptance or rejection of what the client had said, e.g. "Ah", "Right", "indeed", "that's right", "No", "Yeah but". On three occasions there were no markers.

3.3.3 Clarifications. Participants can also interrupt to clarify what has just been said. This happened on 6 occasions (11\%) of shifts.

Example 6 Dialogue C -

1. C: "If I put an SE in and then do an EN it comes up" (C control)

2. E: "So if you put in a ...?" ( $E$ control)

3. C: "SE" (E control)

On two occasions clarifications were prefixed by "now" and twice by "so". On the final two occasions there was no such marker, and a direct question was used.

3.3.4 An explanation of interruptions. We have just described the circumstances in which interruptions occur, but can we now explain why they occur? We suggest the following two principles might account for interrup- 
tions: these principles concern: (a) the information upon which the participants are basing their plans, and (b) the plans themselves.

(A). Information quality: Both expert and client must believe that the information that the expert has about the problem is true and that this information is sufficient to solve the problem. This can be expressed by the following two rules which concern the truth of the information and the ambiguity of the information: (A1) if the speaker believes a fact $P$ and believes that fact to be relevant and either believes that the speaker believes not $P$ or that the speaker does not know $P$ then interrupt; (A2) If the listener believes that the speaker's assertion is relevant but ambiguous then interrupt.

(B). Plan quality: Both expert and client must believe that the plan that the expert has generated is adequate to solve the problem and it must be comprehensible to the client. The two rules which express this principle concern the effectiveness of the plan and the ambiguity of the plan: (B1) If the listener believes $P$ and either believes that $P$ presents an obstacle to the proposed plan or believes that part of the proposed plan has already been satisfied, then interrupt; (B2) If the listener believes that an assertion about the proposed plan is ambiguous, then interrupt.

In this framework, interruptions can be seen as strategies produced by either conversational participant when they perceive that a either principle is not being adhered to.

3.4 Cue reliability. We also investigated whether there were occasions when prompts, repetitions and summaries failed to elicit the control shifts we predicted. We considered two possible types of failure: either the speaker could give a cue and continue or the speaker could give a cue and the listener fail to respond. We found no instances of the first case; although speakers did produce phrases like " $O K^{\prime}$ and then continue, the "OK" was always part of the same intonational contour as that further information and there was no break between the two, suggesting the phrase was a prefix and not a cue. We did, however, find instances of the second case: twice following prompts and once following a summary, there was a long pause, indicating that the speaker was not ready to respond. We conducted a similar analysis for those cue words that have been identified in the literature. Only 21 of the 35 repetitions, summaries and interruptions had cue words associated with them and there were also 19 instances of the cue words "now", "and", "so", "but" and "well" occurring without a control shift.

\section{Control cues and global control}

The analysis so far has been concerned with control shifts where shifts were identified from a series of rules which related utterance type and control. Examination of the dialogues indicated that there seemed to be different types of control shifts: after some shifts there seemed to be a change of topic, whereas for others the topic remained the same. We next went on to examine the relationship between topic shift and the different types of cues and interruptions described earlier. To do this it was necessary first to classify control shifts according to whether they resulted in shifts of topic.

4.1 Identifying topic shifts. We identified topic shifts in the following way: Five judges were presented with the four dialogues and in each of the dialogues we had marked where control shifts occurred. The judges were 
asked to state for each control shift whether it was accompanied by a topic shift. All five judges agreed on 24 of the 56 shifts, and 4 agreed for another 22 of the shifts. Where there was disagreement, the majority judgment was taken.

4.2 Topic shift and type of control shift. Analysing each type of control shift, it is clear that there are differences between the cues used for the topic shift and the no shift cases. For interruptions, $90 \%$ occur within topic, i.e. they do not result in topic shifts. The pattern is not as obvious for prompts and repetitions/summaries, with $57 \%$ of prompts occurring within topic and $67 \%$ of repetitions/summaries occurring within topic. This suggests that change of topic is a carefully negotiated process. The controlling participant signals that he is ready to close the topic by producing either a prompt or a repetition/summary and this may or may not be accepted by the other participant. What is apparent is that it is highly unusual for a participant to seize control and change topic by interruption. It seems that on the majority of occasions (63\%) participants wait for the strongest possible cue (the prompt) before changing topic.

4.3 Other relations between topic and control. We also looked at more general aspects of control within and between topics. We investigated the number of utterances for which each participant was in control and found that there seemed to be organisation in the dialogues above the level of topic. We found that each dialogue could be divided into two parts separated by a topic shift which we labelled the central shift. The two parts of the dialogue were very different in terms of who controlled and initiated each topic. Before the central shift, the client had control for more turns per topic and after it, the expert had control for more turns per topic. The respective numbers of turns client and ex- pert are in control before and after the central shift are :Before 11-7,22-8,12-6,21-6; After 12$33,16-23,2-11,0-5$ for the four dialogues. With the exception of the first topic in Dialogues 1 and 4 , the client has control of more turns in every topic before the central shift, whereas after it, the expert has control for more turns in every topic. In addition we looked at who initiated each topic, i.e. who produced the first utterance of each topic. We found that in each dialogue, the client initiates all the topics before the central shift, whereas the expert initiates the later ones. We also discovered a close relationship between topic initiation and topic dominance. In 19 of the 21 topics, the person who initiated the topic also had control of more turns. As we might expect, the point at which the expert begins to have control over more turns per topic is also the point at which the expert begins to initiate new topics.

\section{Conclusions}

The main result of this exploratory study is the finding that control is a useful parameter for identifying discourse structure. Using this parameter we identified three levels of structure in the dialogues: (a) control phases; (b) topic; and (c) global organisation. For the control phases, we found that three types of utterances (prompts, repetitions and summaries) were consistently used to signal control shifts. For the low level structures we identified, (i.e. control phases), cue words and phrases were not as reliable in predicting shifts. This result challenges the claims of recent discourse theories $(5,10)$ which argue for a the close relation between cue words and discourse structure. We also examined how utterance type related to topic shift and found that few interruptions introduced a new topic. Finally there was evidence for high level structures in these dialogues as evidenced by topic initiation and 
control, with early topics being initiated and dominated by the client and the opposite being true for the later parts.

Another focus of current research has been the modelling of speaker and listener goals ( 1 , 3) but there has been little research on real dialogues investigating how goals are communicated and inferred. This study identifies surface linguistic phenomena which reflect the fact that participants are continuously monitoring their goals. When plans are perceived as succeeding, participants use explicit cues such as prompts, repetitions and summaries to signal their readiness to move to the next stage of the plan. In other cases, where participants perceive obstacles to their goals being achieved, they resort to interruptions and we have tried to make explicit the rules by which they do this.

In addition our methodology is different from other studies because we have attempted to provide an explanation for whole dialogues rather than fragments of dialogues, and used explicit criteria in a bottom-up manner to identify discourse structures. The number of dialogues was small and taken from a single problem domain. It seems likely therefore that some of our findings (e.g the central shift) will be specific to the diagnostic dialogues we studied. Further research applying the same techniques to a broader set of data should establish the generality of the control rules suggested here.

\section{References}

[1] Allen, J.F. and Perrault, C.R. (1980). Analyzing intentions in utterances. Artificial Intelligence, 15, 143-178.

[2] Brennan, S. E., Friedman, M. W., and Pollard, C. (1987) A centering approach to pronouns. In Proceedings of the 25th
Annual Meeting of the Association for Computational Linguistics.

[3] Cohen, P. R. and Levesque, H. J. (1985) Speech acts and rationality. In Proceedings of the 23th Annual Meeting of the Association for Computational Linguistics.

[4] Grosz, B. J., Joshi, A. K., Weinstein, S. (1986) Towards a computational theory of discourse interpretation. Draft.

[5] Grosz, B. J., and Sidner, C. L. (1986) Attentions, intentions and the structure of discourse. Computational Linguistics, 12, $175-204$.

[6] Guindon, R., Sladky, P., Brunner, H., and Conner, J. (1986). The structure of user-adviser dialogues: Is there method in their madness? In Proceedings of the 24th Annual Meeting of the Association for Computational Linguistics.

[7] Hirschberg, J. and Pierrehumbert, J. B. (1986) The intonational structuring of discourse. In Proceedings of the 2/th Annual Meeting of the Association for Computational Linguistics.

[8] Levin, J. A. and Moore, J. A. (1977) Dialogue games: metacommunication structures for natural language interaction. Cognitive Science, 4, 395 - 421.

[9] Polanyi, L. and Scha, R. (1983). Connectedness in Sentence, Discourse and Text. Tilburg University, Tilburg, 141178.

[10] Reichman, R. (1985) Getting computers to talk like you and me. Cambridge, M.A.: MIT Press. 